\title{
Trastorno psicótico delirante, consumo de tóxicos y asesinato con alevosía y ensañamiento
}

\section{Delusional psychotic disorder, consumption of intoxicants and murder with malice aforethought and overkill}

\author{
DOI: $10.46932 / \mathrm{sfjdv2n4-040}$
}

Received in: May 1st, 2021

Accepted in: Jun 30th, 2021

\author{
Dr. Bernat-Noël Tiffon Nonis \\ Universitat Abad Oliba-CEU. Calle de Bellesguard, no30. 08022 Barcelona (Spain). \\ E-mail: btiffonn@uao.es
}

\section{RESUMEN}

Se ilustra un caso de asesinato perpetrado por un sujeto afecto de esquizofrenia paranoide y, por cuyo momento de perpetrarse los hechos, presentaba una crisis psicótica aguda en ausencia de medicación antipsicótico por abandono del tratamiento. Se describe la fenomenología clínico-sintomatológica del perpetrador y del tipo de contenido de pensamiento psicótico-paranoide que mostraba al momento de celebrarse las entrevistas psicológicos-forenses.

Palabras chaves: psicosis, asesinato, impulsividad patológica, alevosía, ensañamiento, trastorno de la personalidad, consumo de tóxicos. cocaina, cannabis, pruebas psicopatologicas.

\begin{abstract}
A case of murder perpetrated by a subject affected by paranoid schizophrenia and, at the time of the perpetration, presenting an acute psychotic crisis in the absence of antipsychotic medication due to treatment abandonment, is illustrated. The clinical-symptomatological phenomenology of the perpetrator and the type of psychotic-paranoid thought content he showed at the time of the psychological-forensic interviews are described.
\end{abstract}

Key words: psychosis, murder, pathological impulsivity, alevosity, murder, personality disorder, toxic use. Cocaine, cannabis, psychopathological testing.

\section{INTRODUCCIÓN:}

El contenido de pensamiento delirante debe contextualizarse dentro de los límites de lo posible, con una coherencia suficiente como para mostrarse aceptable en los trastornos psicopatológicos de naturaleza psicótica (Tiffon, 2021 ${ }^{\mathrm{a}}$ ); siendo que el sujeto procederá de acuerdo con su perturbación (Grimaldi y Bilbao, 2009 en Tiffon, 2021). Asimismo, y con relación a la impulsividad patológica, para Barratt, Stanford, Kent, \& Felthous (1997), la impulsividad es definida como una predisposición a realizar acciones rápidas y no reflexivas en respuesta a estímulos internos y/o externos, a pesar de las posibles consecuencias negativas, tanto para la propia persona como para terceros (en Moeller, Barratt, Dougherty, Schmitz, \& Swann, 2001 en Tiffon y González-Fernándeza , 2021). Según Caviedes y Yonfá (2021) 
existen estudios en los que se menciona la existencia de una comorbilidad entre personalidad paranoide, límite, antisocial e histriónica y el consumo problemático de alcohol (Pérez Moreno et al., 2014; y Prado

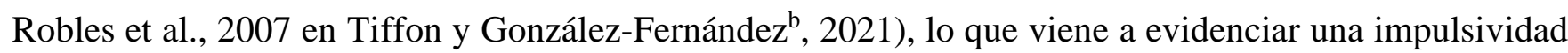
patológica desde la comorbilidad anteriormente mencionada.

\section{EL CASO.}

El presente caso se trata de un varón de 43 años, natural de Barcelona y de estado civil soltero. Se encuentra ingresado en condición de preso preventivo en espera de celebración de juicio en el momento en la que se desarrolla la exploración psicológica. Refiere tener reconocida la Invalidez Permanente en grado de absoluta, desde el año 2007, por Trastorno de la Personalidad Esquizotípico y Evitativo, así como por dependencia al cannabis. Según Ficha Médica del Interno de fecha de septiembre de 2013 del Centro Penitenciario, constan frecuentes visitas psiquiátricas durante los meses de julio, agosto y septiembre.

El informado había ejercido de Vendedor en una Agencia de Viajes (8 años) y como Agente informático (3 años).

El objeto del informe consiste en valorar el estatus psíquico del informado y realizar una aproximación retrospectiva con respecto a los hechos acaecidos en julio de 2013: delito de asesinato de una profesional del sexo acuchillándola con alevosía y ensañamiento (por tanto, sin que la víctima tuviera la oportunidad de defensa y aumentando deliberadamente el dolor de la víctima, respectivamente).

Según la última documentación clínica obrante, consta la cronología del mismo siendo que presenta una "Orientación diagnóstica (DSM-IV): Esquizofrenia paranoide de curso episódico con síntomas interepisódicos residuales. Trastorno por dependencia cannabis/alcohol/cocaína. Constan diagnósticos previos de Trastorno de la Personalidad Esquizoide. Su pronóstico es grave y crónico. Atendiendo al diagnóstico y el curso de la patología no creemos que el paciente se beneficie de un recurso penitenciario ordinario y, en cambio, creemos que pudiera encontrar mejoría de su cuadro clínico en un recurso penitenciario psiquiátrico”.

En otro informe de fecha de julio de 2013, fue visitado psiquiátricamente en el anteriormente citado Centro Penitenciario y en cuya ficha médica se hace constar que el informado sigue: "controles de Psiquiatría desde los 18 años, ideas autorreferenciales, distorsiones psicóticas de la realidad, creencias de poder, leer el pensamiento, creencias místicas (fin del mundo), abandonos del tratamiento. Está con incapacidad absoluta (Trastorno Esquizotípico de la Personalidad, Trastorno de Personalidad por evitación, Fobia Social. Trastorno por adicción al Hachís + Trastorno permanente de personalidad). Ingreso en varios psiquiátricos: Hospital Militar. Se libró del Servicio Militar. Frenopático, hospital de 
día. Temores de que todo el mundo se entere de lo que ha hecho (difusión del pensamiento), referencias de que la TV y la radio hablan de él y que las noticias de los presentadores se referían a él”.

Como hábitos tóxicos destacables en su patobiografía, el informado consume alcohol, sustancias cannabinoides; cocaína, éxtasis, LSD, anfetaminas y Speed.

Con respecto a este último apartado de consumo de tóxicos y tal como según consta en el informe de Servicio de Química del Instituto Nacional de Toxicología y Ciencias Forenses (Ministerio de Justicia), se desprende que “(...) en el cabello analizado se detecta la presencia de cocaína, cocaetileno y cafeína."

La conducta delictivo-criminológica fue el producto de un conjunto de factores psicopatológicos de base que actuaron como condicionantes previos en la materialización conductual anteriormente descrita (Tiffon, $2021^{\mathrm{b}}$ ).

Las siguientes Figuras 1 y 2 muestran los diagramas forenses donde se materializó las fatales e intencionadas puñaladas hacia la víctima (Tiffon, 2017):
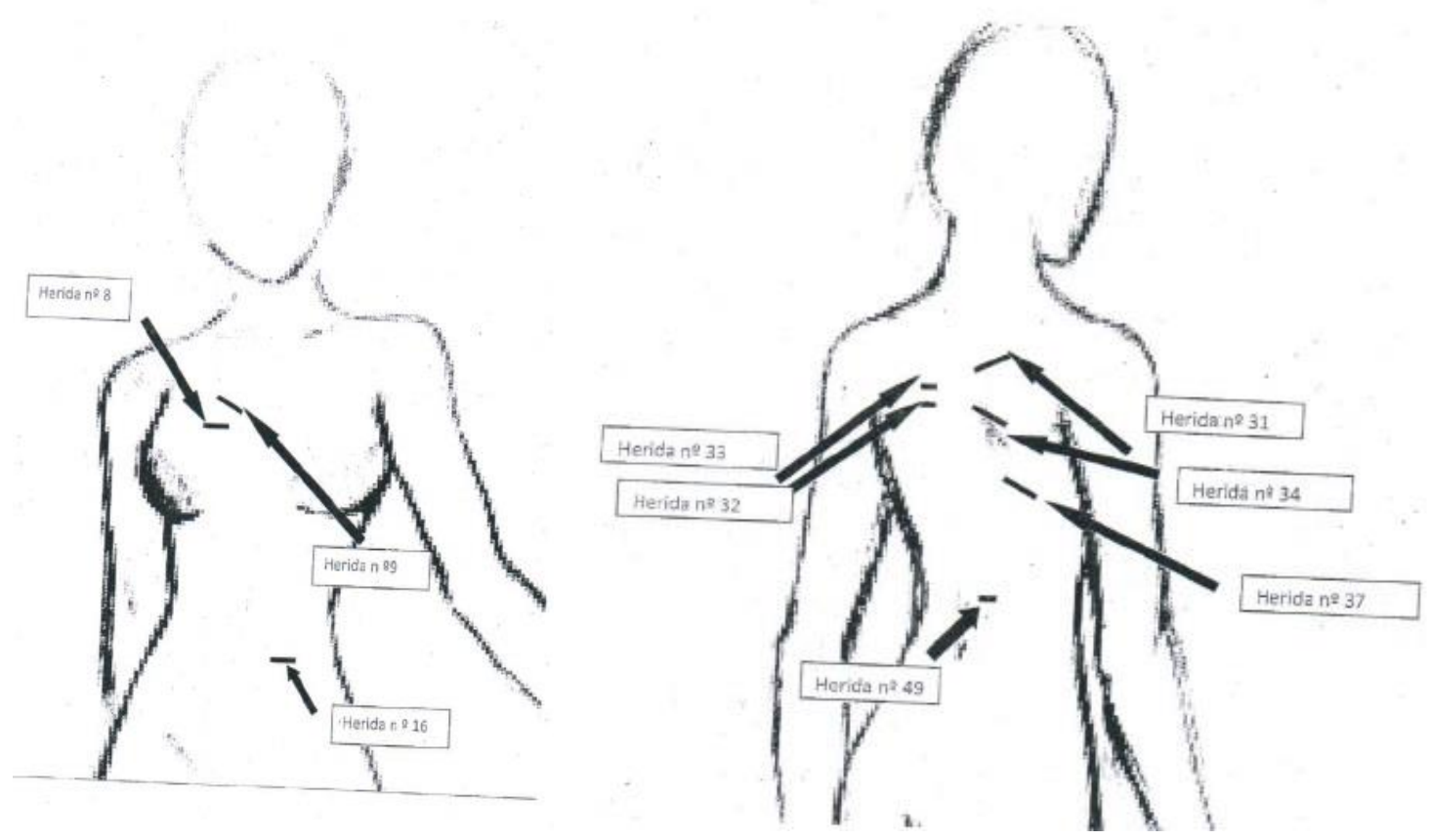

\section{MÉTODO:}

Para la elaboración del presente caso pericial-psicológico/psicopatológico, se ha procedido a la siguiente metodología:

- Entrevista clínico-pericial dirigida (anamnesis) practicada con el informado en el Centro Penitenciario y celebradas en distintas fechas durante el mes de diciembre de 2013 con una suma orientativa y aproximada de horas de visita que asciende de entre unas 16-18 horas.

- Análisis de la documentación clínica: Informe psiquiátrico de la Unidad de Hospitalización Psiquiátrica Penitenciaria (UHPP), Informe Médico-Forense. 
- Evaluación psicopatológica y psicométrica con los siguientes instrumentos de evaluación: MMPI-

2, MCMI-III, Cuestionario Salamanca de Trastornos de Personalidad, Inventario de Evaluación de la Personalidad - PAI, Escala de Hamilton para la Depresión, Inventario de Ansiedad EstadoRasgo, Escala de Impulsividad de Barratt, Test AUDIT - Development of the Alcohol Use Disorders Identification Test-AUDIT-), Escala Multidimensional de Craving de Alcohol [EMCA].

\section{RESULTADOS:}

Los resultados obtenidos de las distintas pruebas administradas son compatibles con la existencia de trastorno de tipo psicótico delirante:

Con respecto al curso y contenido de pensamiento del informado, se desprende la existencia de episodios de pérdida de contacto con la realidad y presencia de pensamiento psicótico-delirante. Refiere que él "escuchaba 5 voces. Esas voces son 5 personalidades de mí mismo. Yo tenía 5 personalidades, cada una con bien y con mal. Eran como espíritus, pero funcionan como personas por sus pensamientos y por su forma de hablar. Las voces me decían que yo era la reencarnación de Lao Tse, de San Juan Bautista, de un señor feudal de Castell de Cabrera de la Anoia, de Montesquieu, de Alejandro Magno, de Alejandro Dumas, del Mago Merlín y de Sigmund Freud. Esas voces ya habían coincidido en otras vidas y éramos un equipo y yo actuaba en representación de este equipo en esta vida. Mi misión era transmitir la Palabra de Dios de manera mesiánica."

Asimismo, también existía un contenido de pensamiento caracterizado por presencia de delirios de tipo místico: “...Mi misión era transmitir la Palabra de Dios de manera mesiánica...”). Un contenido de pensamiento caracterizado por la existencia de "control de pensamiento" ("Caminaba mirando al suelo porque las voces me decían que había alguien con "poderes psíquicos" en la Mafia China dentro de mis ojos y sabían lo que hacía en todo momento y por eso miraba siempre al suelo para que los chinos no supieran ni lo que hacía ni a dónde me dirigía. Los chinos son entes que controlan mi pensamiento y que los tengo dentro de mis ojos y saben en todo momento lo que estoy haciendo. Tengo miedo de los de la Mafia China ya que ellos lo saben todo y me buscan”.

Por último, el informado también refirió que "dentro de sus ojos”, “...existe un ente, de la Mafia China, que saben en todo momento lo que hago y a dónde voy”, “... ellos ven lo mismo que yo veo...”.

\section{DISCUSIÓN:}

El informado, presentaba antecedentes psiquiátricos y psicopatológicos desde la edad de los 18 años, siendo su diagnóstico principal una Esquizofrenia paranoica episódica y con síntomas residuales 
interepisódicos, incluso bajo tratamiento antipsicótico. Patología dual de tipo psicótica-esquizofrénica y de dependencia a tóxicos, todo ello en base de personalidad alterada Clúster A (Esquizotípica, Paranoide y Esquizoide).

Los diagnósticos por el que fue intervenido psicoterapéutica y psicofarmacológicamente fueron los siguientes (documentado mediante historia clínica de orden sanitario público):

- Esquizofrenia paranoide de curso episódico con síntomas interepisódicos residuales.

- Trastorno por dependencia cannabis/alcohol/cocaína

- Trastorno de Personalidad por Evitación (F60.6 del CIE-10).

- Trastorno de la Personalidad Esquizotípico (F21 del CIE-10).

- Trastorno por Dependencia de Cannabis (F19.23 del CIE-10).

- Intoxicación por Alcohol (F10.0 del CIE-10).

Con respecto a los hechos de autos, se hace compatible con que, al momento de perpetrarse los hechos, el informado hubiera padecido una fenomenología clínico-sintomatológica psicótica con pérdida total de contacto con la realidad; considerándose un estado grave de descompensación psicótico-paranoide agravado por el consumo sustancias tóxicas lo que pudiera anular totalmente sus funciones mentales superiores y facultades psíquicas (cognitivas y volitivas). Se ha de señalar que el mismo refiere y relata — aún de forma patológica y sui generis - con relación a los hechos luctuosos anteriormente descritos, debiéndose tener en consideración el dato del abandono del tratamiento psicofarmacológico durante más de seis meses, tal como refirió.

Es por ello que, atendiéndose al diagnóstico clínico y al curso de la psicopatología descrita, se consideró oportuno que el paciente se beneficiase de un recurso penitenciario clínico-psiquiátrico público hospitalario, bajo control estricto psicofarmacológico.

\section{CONCLUSIONES.}

La agudización de los rasgos paranoides ya adolecidos en una estructura de personalidad patológica, la alteración del estado de ánimo y de las emociones de manera repentina y aguda que diera lugar a la explosión conductual impulsiva, súbita e inmediata de una alto grado de agresividad causado por la circunstancia que provocó los hechos, originó sobre el informado que le produjera una disminución de sus capacidades cognitivo-reflexivas, e influyendo nociva y negativamente, en las volitivas de su conducta y de las consecuencias que seguidamente conllevara (Tiffon, 2021 ${ }^{\mathrm{c}}$ ). 
El presente caso viene a ilustrar las dificultades existentes en este tipo de población psicótica por cuya evaluación psicopatológica forense y al que hay que retrotraerse al momento de los hechos luctuosos acaecidos, siempre reviste de una gran dificultad profesional. 


\section{REFERENCIAS}

- $\quad$ Barratt, E. S., Stanford, M. S., Kent, T. A., \& Felthous, A. (1997). Neuropsychological and cognitive psychophysiological substrates of impulsive agression. Biological Psychiatry. 4, 1045-1061.

- $\quad$ Caviedes, G.E.C. \& Yonfá, E.D.A. (2021). “Uso Problemático de Alcohol y Drogas en Mujeres Víctimas de Violencia de Pareja Íntima (VPI)”. Revista Profundidad Psicológica Vol. 1, 2021. Págs. 1-9.

- Grimaldi, E. and Bilbao, G. "El enfermo mental en nuestro ordenamiento jurídico" [Internet]. [Consultation 2009 Jun 10]. Available at: http://servicio.cid.uc.edu.ve/derecho/revista/52/52-3.pdf Extracted on 29.03.2018:

- $\quad$ Moeller, G., Barratt, E., Dougherty, D., Schmitz, J., \& Swann, A. (2001). Psychiatric aspects of impulsivity. American Journal of Psychiatry, 158, 1783-1793.

- $\quad$ Pérez Moreno, J.J., Romero García, M., Salazar Gámez, A., Devesa del Valle, S., Ayala Gambín, J. A., y de Amuedo Rincón, M. (2014). Trastorno Límite de la Personalidad y alcoholismo. Entrevista motivacional: una herramienta para el cambio. Biblioteca Lascasas, 10(3). http://www.indexf.com/lascasas/documentos/lc0796.php

- $\quad$ Prado Robles, V., Crespo Iglesias, JM., Brenlla González, J., y Páramo Fernández, M. (2007). Relación entre consumo de alcohol y rasgos patológicos de personalidad en una muestra de alumnos universitarios. Trastornos Adictivos. 9(2), 126-31. DOI: 10.1016/S1575-0973(07)75638-6.

- $\quad$ Tiffon, B.N. (2015). “Los Crímenes de Perejil”. J.M. Bosch Editor. Barcelona.

- $\quad$ Tiffon, B.-N. (2016). “Archivos Delictivo-Criminológicos”. J.M. Bosch Editor. Barcelona.

- $\quad$ Tiffon, B.-N. (2017). “Atlas de Psicología Forense (Penal)”. J.M. Bosch Editor. Barcelona.

- $\quad$ Tiffon, B.-N. y Cols. (2019). “Atlas Práctico-Criminológico De Psicometría Forense (Volumen I): Asesinatos". J.M. Bosch Editor. Barcelona.

- $\quad$ Tiffon, B.-N. y Cols. (2019). “Atlas Práctico-Criminológico De Psicometría Forense (Volumen II): Tentativas De Asesinatos”. J.M. Bosch Editor. Barcelona.

- $\quad$ Tiffon, B.-N" (2021). "Murder induced deliriously by a "folie à deux". Vol. 2 No. 2 (2021): South Florida Journal of Development, Miami, v. 2, n. 2, apr./jun. 2021 ISSN: 2675-5459 Págs. 3560-3564 Págs. 3560-3564

DOI: https://doi.org/10.46932/sfjdv2n2-191

- $\quad$ Tiffon, B.- $\mathrm{N}^{\mathrm{b}}$ (2021). "Amok syndrome in a quadruple crime, acting out and pathological drive.". Vol. 2 No. 2 (2021): South Florida Journal of Development, Miami, v. 2, n. 2, apr./jun. 2021 ISSN: 26755459 Págs. 3549-3554

DOI: https://doi.org/10.46932/sfjdv2n2-189 
- $\quad$ Tiffon, B.- $\mathrm{N}^{\mathrm{c}}$ (2021). "Criminal personality in a trained elite military and assassination". Vol. 2 No. 2 (2021): South Florida Journal of Development, Miami, v. 2, n. 2, apr./jun. 2021 ISSN: 2675-5459 Págs. 3555-3559.

DOI: https://doi.org/10.46932/sfjdv2n2-190

- $\quad$ Tiffon, B.-N. (2021). "Double filicide for extended suicide (frustrated) of a subject with major psychotic depression and dependent personality disorder". South Florida Journal of Development, Miami, v. 2, n. 3, Special Edition, jul. 2021. Págs. 4552-4562. DOI: https://doi.org/10.46932/sfjdv2n3-057

- $\quad$ Tiffon, B.-N. (2021). "Delusional psychotic disorder, substance use and murder with alevosia and cruelty". South Florida Journal of Development, Miami, v.2, n.3, p.4544-4551 Special Edition, jul. 2021. Págs. 4544-4550. DOI: https://doi.org/10.46932/sfjdv2n3-056

- $\quad$ Tiffon, B.-N. \& González-Fernández ${ }^{a}$ (2021). "Amok Syndrome. Qualified Perspectives on an Aggressive Reaction of Pathological Impulsiveness in The Perpetration of a Double Crime with a Firearm". ASEAN Journal of Psychiatry, Vol. 22(S2), June 2021: 1-5 ISSN: 2231-7805

- $\quad$ Tiffon, B.-N. \& González-Fernández ${ }^{a}$ (2021). "Amok Syndrome. Qualified Perspectives on an Aggressive Reaction of Pathological Impulsiveness in The Perpetration of a Double Crime with a Firearm". ASEAN Journal of Psychiatry, Vol. 22(S2), June 2021: 1-5. ISSN: 2231-7791

- $\quad$ Tiffon, B.-N. \& González-Fernández, J. ${ }^{\text {b }}$ (2021). Alcohol Consumption and Intensification of Paranoid Traits in Attempted Homicide. ASEAN Journal of Psychiatry, Vol. 22(4), June 2021: 1-5. ISSN: $\underline{2231-7805 .}$

- $\quad$ Tiffon, B.-N. \& González-Fernandez, J. ${ }^{\text {b }}$ (2021). Alcohol Consumption and Intensification of Paranoid Traits in Attempted Homicide. ASEAN Journal of Psychiatry, Vol. 22(4), June 2021: 1-5. ISSN: $\underline{2231-7791}$

- $\quad$ Tiffon, B.-N. (2021). "Criminal and Forensic Psychology of a Case of Filicide by Decapitation of a Minor". Journal of Forensic Medicine. Case Series. Volume 6:3. ISSN: 2472-1026 Págs. 1-5 